\title{
Multicavity proton cyclotron accelerator
}

\author{
J. L. Hirshfield* and Changbiao Wang \\ Department of Physics, Yale University, New Haven, Connecticut 06520-8120 \\ V.P. Yakovlev \\ Omega-P, Inc., 199 Whitney Avenue, New Haven, Connecticut 06511
}

(Received 28 January 2002; published 7 August 2002)

\begin{abstract}
A mechanism for acceleration of protons is described, in which energy gain occurs near cyclotron resonance as protons drift through a sequence of rotating-mode $\mathrm{TE}_{111}$ cylindrical cavities in a strong nearly uniform axial magnetic field. Cavity resonance frequencies decrease in sequence from one another with a fixed frequency interval $\Delta f$ between cavities, so that synchronism can be maintained between the rf fields and proton bunches injected at intervals of $1 / \Delta f$. An example is presented in which a $122 \mathrm{~mA}$, $1 \mathrm{MeV}$ proton beam is accelerated to $961 \mathrm{MeV}$ using a cascade of eight cavities in an $8.1 \mathrm{~T}$ magnetic field, with the first cavity resonant at $120 \mathrm{MHz}$ and with $\Delta f=8 \mathrm{MHz}$. Average acceleration gradient exceeds $40 \mathrm{MV} / \mathrm{m}$, average effective shunt impedance is $223 \mathrm{M} \Omega / \mathrm{m}$, but maximum surface field in the cavities does not exceed $7.2 \mathrm{MV} / \mathrm{m}$. These features occur because protons make many orbital turns in each cavity and thus experience acceleration from each cavity field many times. Longitudinal and transverse stability appear to be intrinsic properties of the acceleration mechanism, and an example to illustrate this is presented. This acceleration concept could be developed into a proton accelerator for a high-power neutron spallation source, such as that required for transmutation of nuclear waste or driving a subcritical fission burner, provided a number of significant practical issues can be addressed.
\end{abstract}

DOI: $10.1103 /$ PhysRevSTAB.5.081301

PACS numbers: 96.50.Pw, 14.20.Dh, 41.75.Lx

\section{INTRODUCTION}

Intense proton beams are required in a wide variety of applications. But perhaps the most ambitious proposed project aims to develop $1 \mathrm{GeV}, 45 \mathrm{MW}$ proton accelerators for ATW (Accelerator for Transmutation of Nuclear Waste) [1]. According to Ref. [1], each ATW plant would contain multiple subcritical fission burners driven by two such accelerators, for a total beam power per plant of $90 \mathrm{MW}$. These accelerators would embody superconducting rf technology, with each superconducting linac accelerating a $45 \mathrm{~mA}$ beam to $1 \mathrm{GeV}$ in a length of about $300 \mathrm{~m}$, for an effective acceleration gradient of $3.3 \mathrm{MV} / \mathrm{m}$. The two most powerful proton accelerators in use at present are the $800 \mathrm{MeV}, 1.5 \mathrm{~mA}$ linac at Los Alamos National Laboratory and the $590 \mathrm{MeV}, 1.8 \mathrm{~mA}$ cyclotron at Paul Scherer Institute [2]. Both have much lower beam power than the accelerator discussed here.

This paper describes a new concept for high-efficiency, high-gradient acceleration of a high-current proton beam in a normal conducting (i.e., room temperature) structure; below this conceptual device will be referred to as MPCA (multicavity proton cyclotron accelerator). MPCA consists of a cascade of rotating-mode $\mathrm{TE}_{111}$ cavities in a strong nearly uniform static magnetic field, with cyclotronresonance acceleration in each cavity providing energy gain for the protons. This acceleration mechanism ap-

*Corresponding author.

Email address: jay.hirshfield@yale.edu pears capable of supporting a significantly higher effective acceleration gradient than superconducting rf linacs, higher effective shunt impedances, and much lower peak surface fields in the cavities. The cavity resonance frequencies $f_{n}$ decrease from $f_{1}$ at the first cavity with a fixed frequency interval $\Delta f$ between the $N$ cavities, so that $f_{n}=f_{1}-(n-1) \Delta f$, with $1 \leq n \leq N$. Protons that are injected into the cavity cascade in pulses at time intervals $\Delta T=m / \Delta f$ can experience continuous energy gain, provided the relative phases $\Delta \phi_{n}$ for fields in each of the cavities are properly adjusted; here $m$ is an integer. (Relative phases $\Delta \phi_{n}$ are assigned at $t=0$ and repeat themselves at subsequent time intervals $1 / \Delta f$.) The $\Delta \phi_{n}$ can be adjusted for maximum energy gain, but the values for optimum longitudinal and transverse stability will differ from these, as in an $\mathrm{rf}$ linac. At time intervals of $\Delta T$, the global field pattern in all the cavities reproduces itself so that protons in successive bunches experience identical forces. Since protons can execute a number of orbital turns in each cavity well in excess of unity, voltage gain can be much larger than the product of peak cavity field and cavity length, thus low cavity surface fields can be maintained even for substantial voltage gain. The acceleration mechanism appears to embody dynamic focusing in phase (i.e., longitudinal stability), energy, and beam breadth (i.e., transverse stability), so additional focusing magnets are probably not needed, thereby simplifying the design, conserving space, and not diluting the effective acceleration gradient. Space-charge effects that can limit the beam current in proton cyclotrons should not impose a similar limit in this configuration, since gyrating proton 
orbits do not overlap, and because of the intrinsic dynamic focusing; nevertheless, space-charge effects should be explored to confirm this supposition.

At first glance it might seem that an up-tapered resonant magnetic field $B(z)$ could be used in the MPCA structure to maintain a constant cyclotron frequency $\Omega=e B / m \gamma$ as the protons gain energy and thereby be able to use the same frequency in all the cavities. However, further analysis as described below shows that the resonant magnetic field profile required to maintain resonance will severely limit the acceleration energy that a proton can gain. This energy limit arises since, associated with the axially tapered magnetic field, there must be a transverse magnetic field $\mathbf{B}_{\perp}$, which imparts axial deceleration from the $q \mathbf{v}_{\perp} \times \mathbf{B}_{\perp}$ force, so the axial velocity tends towards zero, i.e., towards stalling. It has been shown that, for the uptapered resonant magnetic field profile which holds constant the resonant phase $\theta=\omega t-\Omega t-k_{z} v_{z} t$ as $\Omega$ and $v_{z}$ change, there is an energy gain limit, given by

$$
(\Delta E)_{\max }=E_{0}\left[\left(\gamma_{0}+1\right) /\left(\gamma_{0}-1\right)\right]^{1 / 2},
$$

where $E_{0}$ is the initial particle kinetic energy and $\gamma_{0}$ is the initial relativistic energy factor [3]. Unlike the microwave cyclotron autoresonance accelerator (CARA) [4] and the laser-driven cyclotron autoresonance accelerator (LACARA) [5], where a relativistic electron beam can experience considerable acceleration before encountering stalling, MPCA employs a proton beam of relatively low initial energy so stalling will occur before protons achieve significant acceleration in an up-tapered resonant magnetic field. When $\gamma_{0}-1 \ll 1$, Eq. (1) gives $(\Delta E)_{\max }[\mathrm{MeV}]=$ $1.37 \sqrt{E_{0}[\mathrm{keV}]}$, so that a $1 \mathrm{MeV}$ proton beam can have a maximum energy gain of $43.3 \mathrm{MeV}$ without stalling. Below we show that $1 \mathrm{MeV}$ protons can be accelerated to 64.6 MeV in the first stage of MPCA with a slowly varied magnetic field and to much higher energy thereafter.

An example is presented below for an eight-cavity cascade in which acceleration of a $122 \mathrm{~mA}$ (average current) proton beam from 1 to $961 \mathrm{MeV}$ in a length of $23.75 \mathrm{~m}$ is predicted. This corresponds to an effective average acceleration gradient of $40.4 \mathrm{MV} / \mathrm{m}$ and an effective overall shunt impedance of $223 \mathrm{M} \Omega / \mathrm{m}$, neglecting short spaces needed between cavities for beyond-cutoff drift tubes and pumping ports. The maximum surface fields in the cavities would range from only 3.6 to $7.2 \mathrm{MV} / \mathrm{m}$. Final average beam power would be over $117 \mathrm{MW}$, in the range needed for ATW. A schematic diagram to illustrate the accelerator concept is shown in Fig. 1.

\section{ANALYSIS}

For preliminary simulation analysis to illustrate the underlying physics of the multicavity proton cyclotron acceleration mechanism, four approximations are made. (i) Distortions of rf field patterns at apertures between cavities are assumed to be negligible, since the proton gyration radius and aperture radius are considerably smaller than the cavity radius, as shown in Fig. 1. (ii) The proton energy increase per rf cycle in each cavity is taken equal to the cavity stored energy multiplied by $2 \pi\left(1 / Q_{L}-\right.$ $\left.1 / Q_{o}\right) \ll 1$, since rapid variations of field amplitude due to pulsed beam loading are strongly damped by the long cavity response time; here $Q_{L}$ and $Q_{o}$ are the cavity quality factors with beam loading and without. (iii) Spacecharge effects are neglected, since proton bunches are spread out over part of the orbit circumference, and successive bunches cannot overlap. (iv) The injected beam is taken in the example discussed to have zero emittance. These simplifications allow use of single-particle equations of motion to characterize proton acceleration in an idealized MPCA. The results obtained obviously cannot account for certain practical aspects of an actual accelerator, including realistic cavity fields, influence of space charge, and injector requirements.

The electromagnetic fields in cylindrical coordinates $(r, \theta, z)$ for $\mathrm{TE}_{111}$ rotating modes in a simple cylinder of radius $R$ and length $L$, together with the weakly nonuniform applied static magnetic field $B_{o}(z)$, are given by

$$
B_{z}=\hat{B} J_{1}\left(k_{c} r\right) \sin (\pi z / L) \sin (\omega t-\theta)+B_{o}(z),
$$

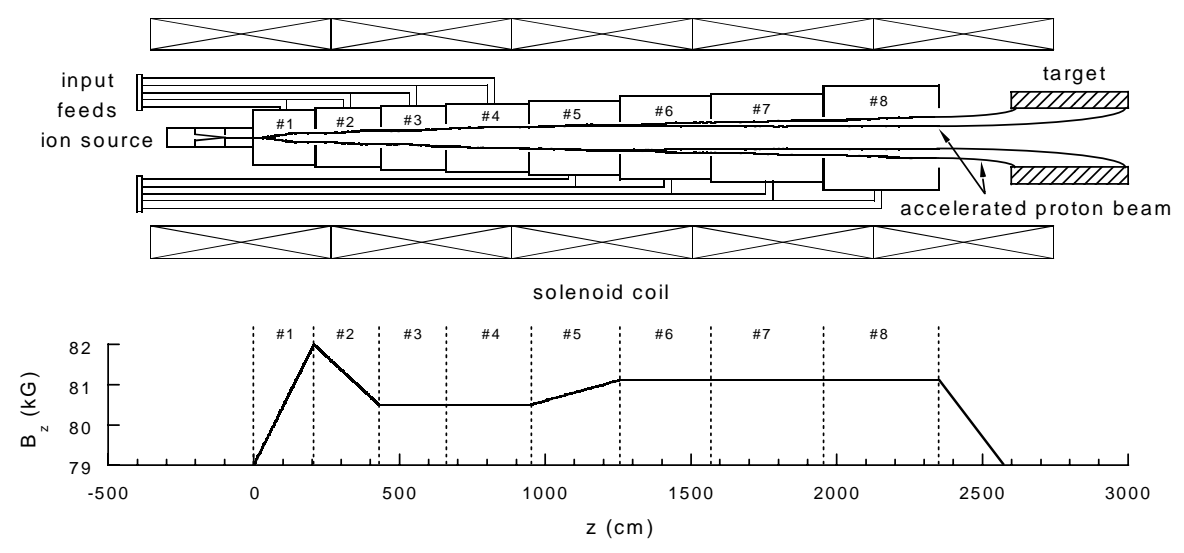

FIG. 1. Schematic of the multicavity proton cyclotron accelerator. 


$$
B_{r}=\hat{B} \frac{\pi}{k_{c} L} J_{1}^{\prime}\left(k_{c} r\right) \cos (\pi z / L) \sin (\omega t-\theta)-\frac{r}{2} \frac{d B_{o}}{d z}
$$

$$
B_{\theta}=-\hat{B} \frac{\pi}{k_{c} L} \frac{1}{k_{c} r} J_{1}^{\prime}\left(k_{c} r\right) \cos (\pi z / L) \cos (\omega t-\theta)
$$

$$
\begin{gathered}
E_{z}=0, \\
E_{r}=-\hat{B} \frac{\omega}{k_{c}} \frac{1}{k_{c} r} J_{1}\left(k_{c} r\right) \sin (\pi z / L) \sin (\omega t-\theta),
\end{gathered}
$$

and

$$
E_{\theta}=\hat{B} \frac{\omega}{k_{c}} J_{1}^{\prime}\left(k_{c} r\right) \sin (\pi z / L) \cos (\omega t-\theta),
$$

where $\omega$ is the cavity operating frequency, $J_{1}(x)$ is the firstorder Bessel function, and $k_{c}=j_{11}^{\prime} / R$ with $j_{11}^{\prime}$ the first zero of the Bessel function derivative. Energy conservation is invoked to find the rf magnetic field amplitude $\hat{B}$ in each cavity and average beam current $I$, namely,

$$
\hat{B}=\left[\frac{1}{\varepsilon_{0} \pi R^{2} L} \frac{k_{c}^{2}}{\omega^{3}} \frac{4 Q_{L} P_{\mathrm{in}}}{J_{1}^{2}\left(j_{11}^{\prime}\right)\left(1-1 / j_{11}^{\prime 2}\right)}\right]^{1 / 2},
$$

and

$$
I=\left(1-\frac{Q_{L}}{Q_{0}}\right) \frac{q_{p} P_{\text {in }}}{m_{p} c^{2}\left(\left\langle\gamma_{f}\right\rangle-1\right)},
$$

where $P_{\text {in }}$ is the input rf power, $q_{p}$ is the proton charge, $m_{p}$ is the proton rest mass, and $\left\langle\gamma_{f}\right\rangle$ is the beam-averaged relativistic energy factor at the end of the cavity. (Spreads occur in beam parameters due to a finite-phase-width injection window and a finite initial beam emittance.) The interaction of protons with the electromagnetic fields is calculated using the single-particle equations of motion cavity by cavity with $P_{\text {in }}$ and $Q_{L}$ iterated to yield the fixed current value $I$ according to Eq. (9). The relative phases between the rf fields in the cavities are adjusted to best increase proton energy gain, while limiting the spreads in energy and phase; low phase spread after one cavity allows greater acceleration in the next. It was found that the use of 16 computational particles distributed uniformly over the injection phase window is sufficient for obtaining accurate computations. Results of preliminary calculations for this accelerator concept have been presented at conferences and workshops $[3,6,7]$, but none of those prior examples showed effective acceleration gradients $>20 \mathrm{MV} / \mathrm{m}$, nor achieved final proton energies $>530 \mathrm{MeV}$. Results shown below are the first obtained for parameters that might apply for a high-power neutron spallation source suitable for ATW.

\section{EXAMPLE}

For an illustrative preliminary example, we employ a cascade of eight $\mathrm{TE}_{111}$ simple cylindrical cavities with $\Delta f=8 \mathrm{MHz}$ and with resonant frequencies of 120 , $112, \ldots$, and $64 \mathrm{MHz}$. Thus 15, 14,.., and 8 full rf cycles pass in these cavities, respectively, between proton bunches if they are injected every $125 \mathrm{~ns}$ (i.e., at an $8 \mathrm{MHz}$ rate). Cumulative acceleration can occur equally for each bunch because the global field pattern throughout the entire cavity cascade reconstructs itself exactly each $125 \mathrm{nsec}$. Average beam current is chosen to be $122 \mathrm{~mA}$, injected in $2.5 \mathrm{nsec}$ bunches (30\% of an rf cycle) and thus with peak currents of $6.1 \mathrm{~A}$ (duty factor $=1 / 50$ ); the energy of the injected protons is taken to be $1 \mathrm{MeV}$. The static magnetic field traversing the cavities varies in the range 7.9-8.2 T, with a gentle taper in magnetic field introduced at cavity No. 1, cavity No. 2, and cavity No. 5 to increase the energy gain. Parameters for each of the eight stages are given in Table I. The intrinsic (Ohmic) quality factors $Q_{o}$ for the cavities are calculated assuming fabrication with copper and an operating temperature of $20^{\circ} \mathrm{C}$. Final beam power is $117.1 \mathrm{MW}$, rf-to-beam power efficiency is $67.3 \%$, and average effective acceleration gradient is $40.4 \mathrm{MV} / \mathrm{m}$. rf power density to be dissipated on the cavity walls averages $21.2 \mathrm{~W} / \mathrm{cm}^{2}$, and has a peak value of $113.5 \mathrm{~W} / \mathrm{cm}^{2}$ in the center of the cylindrical wall of cavity No. 1, but has peak values at the other cavities that fall from 53 to $22 \mathrm{~W} / \mathrm{cm}^{2}$.

TABLE I. Parameters for an eight-cavity proton accelerator with $122 \mathrm{~mA}$ average current.

\begin{tabular}{ccccccccc}
\hline \hline $\begin{array}{c}\text { Stage } \\
\text { No. }\end{array}$ & $\begin{array}{c}\text { Cavity } \\
\text { frequency } \\
(\mathrm{MHz})\end{array}$ & $\begin{array}{c}\text { Cavity } \\
\text { radius } \\
(\mathrm{cm})\end{array}$ & $\begin{array}{c}\text { Cavity } \\
\text { length } \\
(\mathrm{m})\end{array}$ & $\begin{array}{c}\text { rf power } \\
\text { input } \\
(\mathrm{MW})\end{array}$ & $\begin{array}{c}\text { Beam-loaded } \\
\text { cavity } \\
Q_{L}\end{array}$ & $\begin{array}{c}\text { Intrinsic } \\
\text { cavity } Q_{o} \\
\text { (copper) }\end{array}$ & $\begin{array}{c}\text { Peak surface } \\
\text { field } \\
(\mathrm{MV} / \mathrm{m})\end{array}$ & $\begin{array}{c}\text { Mean final } \\
\text { energy gain } \\
(\mathrm{MeV})\end{array}$ \\
\hline 1 & 120 & 92 & 2.06 & 18.0 & $6.25 \times 10^{4}$ & $1.1 \times 10^{5}$ & 7.2 & 63.6 \\
2 & 112 & 98 & 2.23 & 15.0 & $3.23 \times 10^{4}$ & $1.1 \times 10^{5}$ & 4.4 & 86.8 \\
3 & 104 & 106 & 2.39 & 15.5 & $4.72 \times 10^{4}$ & $1.2 \times 10^{5}$ & 5.0 & 76.9 \\
4 & 96 & 110 & 2.81 & 18.5 & $4.50 \times 10^{4}$ & $1.2 \times 10^{5}$ & 4.9 & 94.7 \\
5 & 88 & 120 & 3.07 & 24.0 & $4.13 \times 10^{4}$ & $1.2 \times 10^{5}$ & 4.9 & 128.7 \\
6 & 80 & 132 & 3.38 & 23.0 & $3.35 \times 10^{4}$ & $1.3 \times 10^{5}$ & 4.0 & 139.9 \\
7 & 72 & 144 & 3.92 & 30.0 & $3.55 \times 10^{4}$ & $1.3 \times 10^{5}$ & 4.2 & 178.4 \\
8 & 64 & 172 & 3.89 & 30.0 & $3.34 \times 10^{4}$ & $1.5 \times 10^{5}$ & 3.6 & 190.7 \\
Total & & & 23.75 & 174.0 & & & & 959.7 \\
\hline \hline
\end{tabular}




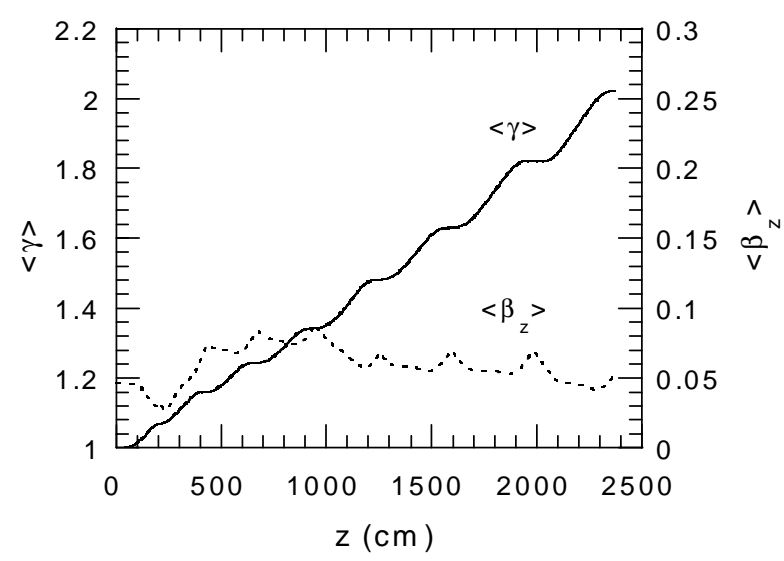

FIG. 2. Average energy factor $\langle\gamma\rangle$ and average normalized axial velocity $\left\langle\beta_{z}\right\rangle$ vs $z$.

In Fig. 2 are shown the progressions along the accelerator axis $z$ of average energy factor $\langle\gamma\rangle$, and average axial proton velocity normalized to the speed of light $\left\langle\beta_{z}\right\rangle$. As noted above, no space between cavities is reserved for necessary beyond-cutoff drift tubes or for vacuum pumping. But it is expected that the drift tubes will not need to be very long, since the electric field is already zero at the cavity end walls, and since the $\mathrm{TE}_{11}$ mode is the lowest propagating mode. It is furthermore assumed that coaxial input couplers feed power through the outer cylindrical walls of the cavities and thus require no longitudinal space; at least two cavity inputs disposed in azimuth at $90^{\circ}$ and excited in time quadrature are needed to realize field rotation, but more may be needed to handle the high-power levels. In Fig. 3 is shown the path for transverse excursions of a typical proton, as projected onto the $x-z$ plane. Protons execute $20,20,11,12,13,15,16$, and 18 turns in the eight cavities, respectively. After the eighth cavity the magnetic field can be tapered down, thus causing the proton orbits to fan out so as to intersect a neutron spallation target. Self-scanning of the beam, and careful design of the shape of the field taper, would allow the $117 \mathrm{MW}$ beam power to be distributed over a large enough target area for orderly dissipation of the power. In

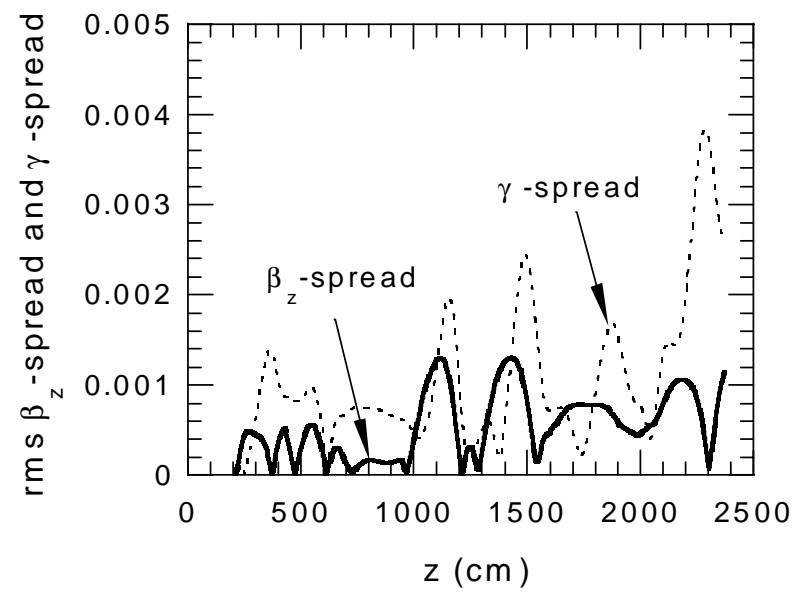

FIG. 4. rms spreads in proton energy factor and axial velocity along the accelerator. These spreads are nearly zero in the first cavity, since the injected beam is taken to have zero emittance. The oscillatory character of these spreads is evidence of dynamic (transverse) stabilization.

Fig. 4 are shown the progressions in rms spreads in proton energy and normalized axial velocity along the accelerator axis, while Fig. 5 shows the rms spread in resonance phase (the difference between proton gyration phase and rf field phase). At the end of the eighth cavity, these spreads are seen to be $2.44 \mathrm{MeV}(0.25 \%), 0.0012$, and $0.107 \mathrm{rad}$, respectively. The fact that these spreads exhibit oscillations along the acceleration path is strong evidence for the intrinsic stability of this acceleration mechanism. Since the injected proton beam is taken to have zero emittance, the final energy and momentum spreads are attributable only to the finite-length injection phase window. This window is $2.5 \mathrm{~ns}$ in the present example (30\% of an rf cycle in the first cavity); in general, a larger injection phase window will result in larger spreads.

The radii of the $\mathrm{TE}_{111}$ simple cylindrical cavities are seen in Fig. 3 and Table I to be significantly larger than the orbit radius of the protons. Smaller radii cavities can probably be designed that have the necessary apertures, using radial vanes and/or tuned quarter-wave stubs. Thus the solenoid magnet can — in practice — probably have smaller

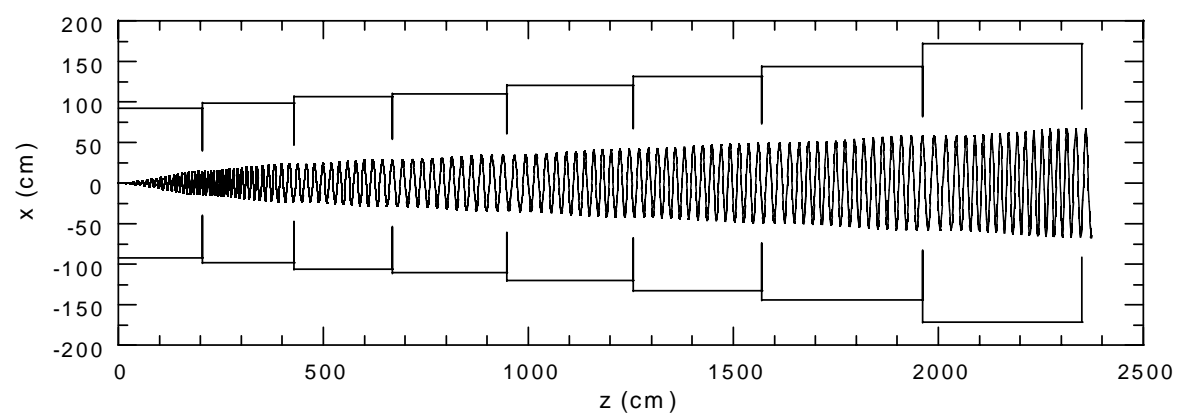

FIG. 3. Projection onto the $x-z$ plane of the orbit of a typical proton. Outlines of the cavities are also shown. Since the orbit radii are considerably smaller than the cavity radii, the opportunity exists to insert radial vanes that will result in smaller outer radii for the cavities while preserving adequate apertures for the beam. 


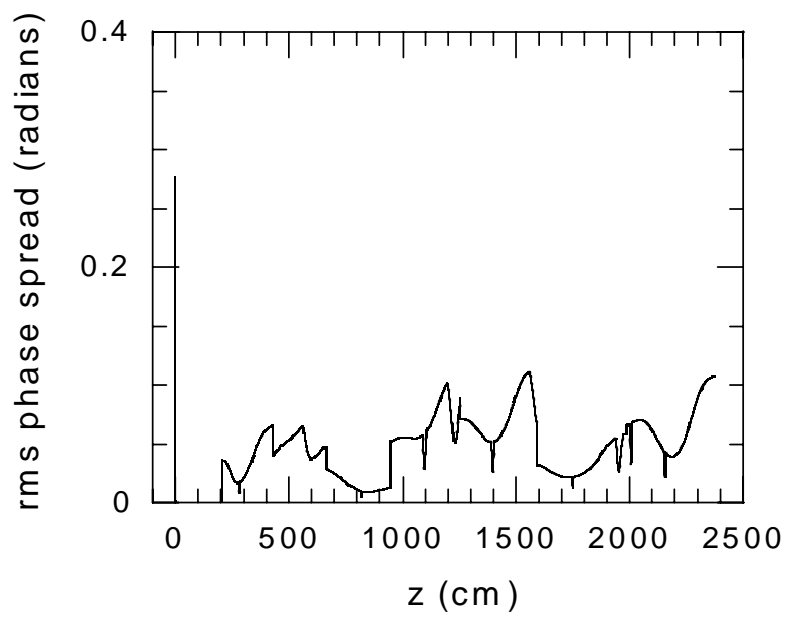

FIG. 5. rms spread in resonance phase along the accelerator. The phase spread in the first cavity drops rapidly from $0.28 \mathrm{rad}$ to nearly zero, since the injected beam is taken to have zero emittance. The oscillatory character of this spread is evidence of dynamic (longitudinal) stabilization.

radius than is implied from the cavity radii as listed in Table I.

\section{PHASE FOCUSING}

In Figs. 4 and 5, it is seen that the rms spreads in proton energy, axial velocity, and resonance phase all oscillate and remain bounded during the acceleration process. As stated above, this is evidence of inherent longitudinal and transverse stability. Further insight into the intrinsic stability of the acceleration process can be found by examining proton dynamics in the first two stages of the accelerator. The same parameters as in the example discussed above are used, except that the relative phase $\phi_{2}-\phi_{1}$ is adjusted not for maximum energy gain $(0.90 \pi \mathrm{rad}$ as in the example above) but for $0.68 \pi \mathrm{rad}$ - the value for minimum bunch length. Figure 6 shows a transverse portrait of the loci of 400 computational particles in the plane $z=429 \mathrm{~cm}$ (end of second cavity) which all arrive during a time interval of $0.5 \mathrm{nsec}$, after having been injected into the first cavity during a time interval of $2.5 \mathrm{nsec}$. Figure 6 also shows the loci at $z=206 \mathrm{~cm}$ (end of first cavity) showing - between the entry and exit of the second cavity - a reduction by a factor of $\sim 13$ in the bunch length measured along the orbit and a reduction of a factor of 5 in the bunch time. (These factors differ because the particles are traveling on steep helical orbits.)

The intrinsic phase focusing evident in Fig. 6 suggests that additional focusing is probably not needed for the proton beam to remain confined during acceleration, and that the intrinsic focusing forces could serve to control space charge (which is not included in the analysis presented here).

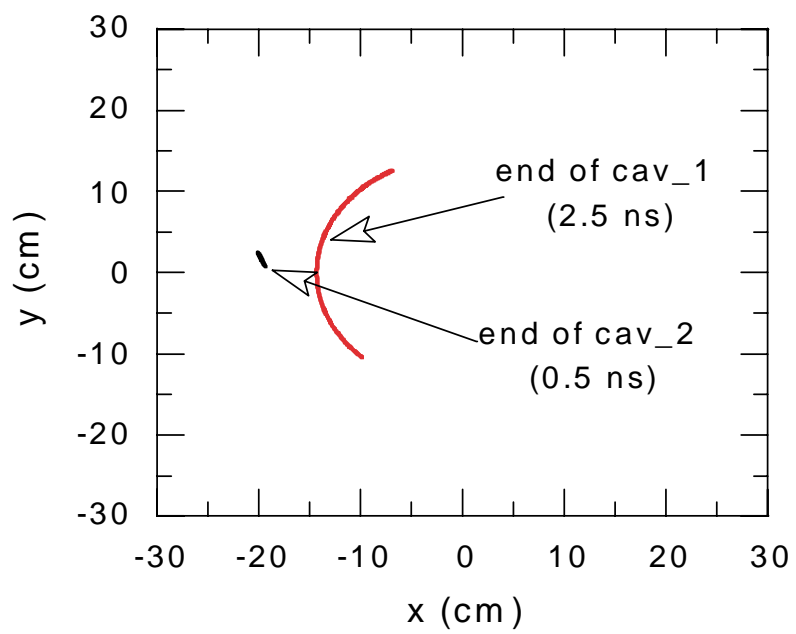

FIG. 6. (Color) Transverse portrait of the loci of 400 computational particles in the planes $z=206 \mathrm{~cm}$ (end of first cavity) and $z=429 \mathrm{~cm}$ (end of second cavity). All the particles arrive at the end of the first cavity during a time interval of $2.5 \mathrm{nsec}$ and at the end of the second cavity during a time interval of $0.5 \mathrm{nsec}$, showing a reduction by a factor of $\sim 13$ in the bunch length and a reduction of a factor of 5 in the arrival time.

\section{DISCUSSION}

The preliminary computational results shown here suggest that - in principle - a high intensity proton accelerator in the $1 \mathrm{GeV} / 100 \mathrm{MW}$ range might be built with important practical distinctions, as compared with accelerators based on superconducting linac designs. These distinctions include (i) high effective acceleration gradient ( $>40 \mathrm{MV} / \mathrm{m}$ in the example shown), (ii) apparent intrinsic phase (longitudinal) and energy (transverse) dynamic orbit stability, (iii) high effective shunt impedance $(223 \mathrm{M} \Omega / \mathrm{m}$ in the example shown), (iv) low surface electric fields in the cavities $(<7.2 \mathrm{MV} / \mathrm{m}$ in the example shown), (v) low rms energy spread for the accelerated beam $(0.25 \%$ in the example shown), (vi) large aperture in the cavities to help avoid beam interception, and (vii) relaxed space-charge limitations on beam current since proton orbits do not intersect and because of the aforementioned dynamic phase focusing.

Against these positive distinctions it is necessary to balance the need for a large superconducting solenoid magnet: the $8 \mathrm{~T}$ magnet taken in the example is evidently well within the present state of the art, but would undoubtedly be costly. The required magnet radius could be smaller than the cavity radii listed in Table I, once cavity designs invoking vanes or other internal features are perfected [6]. It can be shown that vaned cavities can have shunt impedances similar to those of simple cylinders [8]. Cavity frequencies scale in direct proportion to magnetic field strength, so availability of a field stronger than the $8 \mathrm{~T}$ could further reduce the radius and length of the machine. 
In any case, Table I shows that the required apertures are much smaller than the radii for the simple cylindrical cavities used in the example, so that design of internal structures to reduce overall cavity radii could lead to distinct reductions in machine and magnet radius. It is also possible that judicious cavity design can result in higher rf-tobeam efficiency $\eta$ than was found for cylindrical cavities.

The magnet cost, together with other costs for this system, will need to be eventually considered in comparing the multicavity proton cyclotron with, for example, the cost of a pair of superconducting linacs with a lower acceleration gradient. A second item to balance against the above positive distinctions is the less-than-ideal efficiency $\eta$ engendered by the use of room-temperature cavities ( $\eta=67 \%$ in the example given). In the simulations presented here, space-charge and finite beam emittance effects are neglected. For an ideal continuous solid beam, the full voltage depression caused by space charge is given by $30 I\left[1+2 \ln \left(R_{w} / r_{b}\right)\right] / \beta_{z}$ [9], with $I$ the beam current, $R_{w}$ the wall radius, and $r_{b}$ the beam radius. With $r_{b}=1 \mathrm{~cm}$ and the other parameters as used in the above example, one finds the voltage depression to be $40 \mathrm{kV}$, much less than the injected beam voltage $1 \mathrm{MV}$. For a short pulse beam, as in the example, the voltage depression is probably smaller. However, to better understand the role of space charge in the MPCA, more sophisticated computations are needed. Furthermore, detailed engineering studies must be undertaken on such items as the ion source and injector for generating high peak current proton beams, the rf power distribution system, cavity cooling, beam control between cavities, and target interactions for a diverging beam exiting MPCA. Nevertheless, given the attributes of the new proton accelerator concept presented in this paper, such further study would seem justified so as to allow an informed choice to be made in the final selection of the accelerator to be built for a spallation source for ATW.

\section{ACKNOWLEDGMENTS}

Constructive discussions were held with O.A. Nezhevenko and R. S. Symons.

[1] G. Lawrence, T. Wangler, S. Schriber, K. Shepard, P. Wanderer, and M. Allen, LANL Report No. LA-UR-99-3225, 1999.

[2] H. Lengeler, in Handbook of Accelerator Physics and Engineering, edited by A. W. Chu and M. Tigner (World Scientific, Singapore, 1999), p. 40.

[3] J. L. Hirshfield, C. Wang, and R. Symons, in Proceedings of the ANS Winter Meeting, Reno, Nevada, 2001 (to be published).

[4] C. Wang and J. L. Hirshfield, Phys. Rev. E 51, 2456 (1995).

[5] J. L. Hirshfield and C. Wang, Phys. Rev. E 61, 7252 (2000).

[6] J. L. Hirshfield, C. Wang, and R. Symons, in Advanced Accelerator Concepts: Ninth Workshop, edited by P.L. Colestock and S. Kelley, AIP Conf. Proc. No. 569 (AIP, New York, 2001), p. 833.

[7] C. Wang and J.L. Hirshfield, in Proceedings of the Particle Accelerator Conference, 2001, edited by P. Lucas and S. Webber (IEEE, Piscataway, NJ, 2001), p. 3389.

[8] V.P. Yakovlev (private communication).

[9] M. Reiser, Theory and Design of Charged Particle Beams (Wiley, New York, 1994), p. 194. 